\section{Erblast gering \\ Gesunder Nachwuchs trotz Strahlentherapie}

Das Risiko ein Kind mit genetischem Schaden zu bekommen, ist bei Paaren, bei denen ein Partner in früheren Jahren aufgrund einer Krebserkrankung chemo- oder strahlentherapeutisch behandelt wurde, nur um 0,2\% erhöht. Das zeigten die Ergebnisse der Arbeitsgruppe um Jeanette Winther von der dänischen Krebsgesellschaft. Sie hatten die Daten von 1.474 Überlebenden - 752 Frauen und 722 Männern - aus dem nationalen Krebsregister ausgewertet, die vor dem 20. Lebensjahr an Krebs erkrankt waren und Kinder bekommen hatten (insgesamt 2.767). Von 1.020 analysierten Schwangerschaften hatten 156 Kinder einen genetischen Defekt, 864 Kinder keinen. Definiert waren als solche Defekte chromosomale Aberrationen, angeborene Missbildungen, Totgeburten und frühkindliche Todesfälle. Verglichen wurden jeweils die Risiken bestrahlter vs. nicht bestrahlter Eltern bzw. chemotherapierter vs. nicht chemotherapierter Eltern.

Einen deutlichen, wenn auch nicht signifikanten Einfluss $(p=0,07)$ auf angeborene Missbildungen, Totgeburten und frühkindlichen Tod zusammen

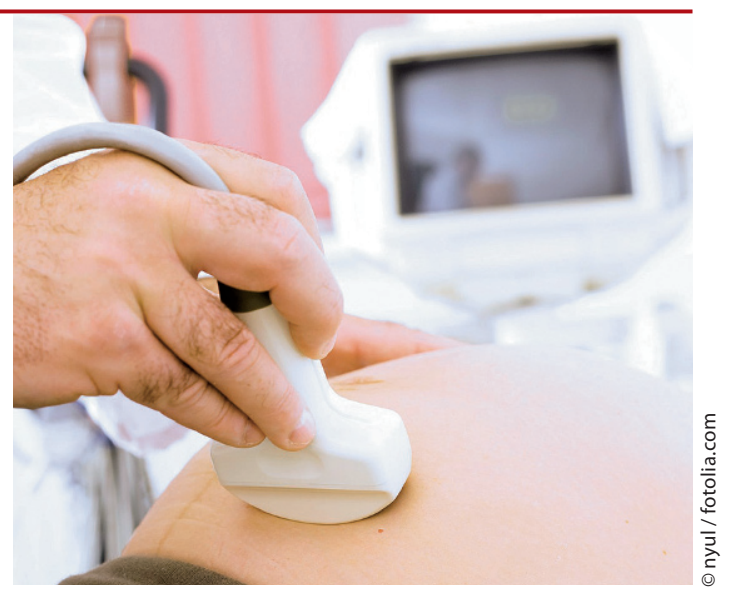

Große Sorge ehemaliger Krebspatientinnen: Wird mein Baby gesund zur Welt kommen?

genommen hatte die Dosis, die die Gebärmutter bei Bestrahlungen von Unterbauch und Becken abbekommen hatte. So war die höchste Dosis mit einem verdoppelten Risiko eines solchen Ereignisses verbunden. Weder die Bestrahlung der Ovarien bei Mädchen noch der Hoden bei Jungen schien das spätere Risiko für den Nachwuchs zu beeinflussen. Dabei hatten die Strahlendosen teilweise nur knapp unter der Unfruchtbarkeitsschwelle gelegen.

Erwähnenswert außerdem: Unter den Kindern fand sich kein einziger Fall von Leukämie.

\section{Elke Oberhofer}

Winther JF et al. Genetic Disease in the Children of Danish Survivors of Childhood and Adolescent Cancer. J Clin Oncol. 2011;30(1):27-33

\title{
Operiertes Pankreaskarzinom Noch kein Standard für die Nachsorge
}

Derzeit existieren sehr unterschiedliche Empfehlungen für die postoperative Überwachung von $\mathrm{Pa}-$ tienten mit Pankreaskarzinom. Amerikanische Forscher untersuchten nun anhand populationsbasierter Daten, wie Patienten im Anschluss an eine Resektion in kurativer Absicht nachbeobachtet wurden. Bei 2.393 Patienten mit Pankreaskarzinom beurteilten sie über einen Zeitraum von zwei Jahren den postoperativen Verlauf. Beurteilt wurden Parameter wie Arztbesuch und Computertomografie (CT) in vier verschiedenen Zeitfenstern. Ausschlusskriterien waren Tod, Rezidiv oder Aufnahme in ein Hospiz. Insgesamt betrug die mittlere Überlebenszeit 14,6 Monate.

Die Auswertung der Daten erbrachte, dass es keine einheitliche Nachsorgepraxis gibt. So erhielten etwa im zweiten Beobachtungsjahr $42 \%$ der Überlebenden kein CT, während bei $27 \%$ gleich mehrere Aufnahmen gemacht wurden. Bei 20,9\% der Patienten wurde im vierten Beobachtungsmonat ein CT durchgeführt, im 27. Monat war der Anteil auf 6,4\% gesunken. Eine Regelmäßigkeit (z. B. CT-Kontrolle alle sechs Monate) ließ sich aus den Daten nicht erkennen. Besonders häufig kontrolliert wurden $\mathrm{Pa}$ tienten mit adjuvanter Chemo- oder Radiotherapie $(58,4 \%)$ oder solche, bei denen die Diagnose erst im fortgeschrittenen Alter gestellt worden war.

Die Studie verdeutlicht die dringende Notwendigkeit, Nachsorgeprotokolle in der postoperativen Versorgung von Patienten mit Pankreaskarzinom zu evaluieren und zu standardisieren. Besonders hinsichtlich der erforderlichen CTKontrollen bestehen offenbar große Unsicherheiten. Da bisher keine evidenzbasierten Leitlinien vorliegen, empfehlen die Autoren, entsprechend den Vorschlägen des National Comprehensive Cancer Network (NCCN), bis zu zwei Jahre postoperativ alle drei bis sechs Monate ein CT durchzuführen.

\section{Christine Starostzik}

Sheffield KM et al. Surveillance of pancreatic cancer patients after surgical resection. Ann Surg Oncol. 2011; Dec 6. [Epub ahead of print]. 